\title{
Pacific
}

Journal of

Mathematics

\section{AVERAGING SEQUENCES}

Fernando Alcalde Cuesta And Ana Rechtman

Volume $255 \quad$ No. 1

January 2012 


\title{
AVERAGING SEQUENCES
}

\author{
Fernando Alcalde Cuesta And AnA ReCHTMan
}

\begin{abstract}
In the spirit of the Goodman-Plante average condition for the existence of a transverse invariant measure for foliations, we give an averaging condition to find tangentially smooth measures with prescribed Radon-Nikodým cocycle. Harmonic measures are examples of tangentially smooth measures for foliations and laminations. We also present a sufficient hypothesis under which the tangentially smooth measure is harmonic.
\end{abstract}

\section{Introduction}

Averaging sequences for foliations were introduced in the pioneering work of Plante [1975] on the influence that the existence of transverse invariant measures exerts on the structure of a foliation. Although that work dealt only with the case of subexponential growth, his approach is clearly reminiscent of the classic work of E. Følner [1955] on groups. Using the same kind of ideas, S. E. Goodman and Plante [1979] exhibited an averaging condition which guarantees the existence of transverse invariant measures for foliations of compact manifolds.

In this paper we formulate a more general averaging condition which gives rise to a tangentially smooth measure for a compact laminated space $(M, \mathscr{F})$. This condition may be related to the $\eta$-Følner condition in [Alcalde Cuesta and Rechtman 2011], in the same spirit as Følner, but using a modified Riemannian metric along the leaves. The modification is done by replacing any complete Riemannian metric along the leaves with the product of the metric with some density function. Namely, given a compact laminated space and a positive cocycle defined on the equivalence relation induced by the lamination on a total transversal, we prove that an $\eta$-Følner sequence gives rise to the existence of a tangentially smooth measure whose Radon-Nikodým cocycle is the given one. Moreover, we describe a sufficient hypothesis for obtaining a harmonic measure. This is the content of Theorem 4.10.

This work was partially supported by the Consejo Nacional de Ciencia y Tecnología (CONACyT) in Mexico and the Xunta de Galicia INCITE09E2R207023ES in Spain.

MSC2010: 37A20, 43A07, 57R30.

Keywords: lamination, discrete equivalence relation, measure. 
Before proving Theorem 4.10, we analyze the discrete case. We define an averaging condition for any equivalence relation $\mathscr{R}$ defined by a finitely generated pseudogroup acting on a compact space and any continuous cocycle $\delta: \mathscr{R} \rightarrow \mathbb{R}_{+}^{*}$ that we call a $\delta$-averaging condition. In Theorem 3.6 we prove that the existence of a $\delta$ averaging sequence gives a quasi-invariant measure with Radon-Nikodým cocycle $\delta$. Under some additional conditions, in particular if $\delta$ is harmonic, the measure obtained is harmonic. In this case, our result is reminiscent of Kaimanovich's [1997] characterization of amenable equivalence relations.

The paper is organized as follows. In Section 2 we review some preliminaries. In particular Section 2C contains the proof of Goodman and Plante's theorem. The discussion of the discrete and continuous settings is split into two separate sections, Section 3 and Section 4, respectively, which can be read independently. In Section 5 we analyze some explicit examples. The relation between the two types of averaging sequences will be briefly discussed in Section 6 .

\section{Preliminaries}

2A. Laminations and equivalence relations. A compact space $M$ admits a $d$ dimensional lamination $\mathscr{F}$ of class $C^{r}$ with $1 \leq r \leq \infty$ if there exists a cover of $M$ by open sets $U_{i}$ homeomorphic to the product of an open disc $P_{i}$ in $\mathbb{R}^{d}$ centered at the origin and a locally compact separable metrizable space $T_{i}$. Thus, if we denote the corresponding foliated chart by $\varphi_{i}: U_{i} \rightarrow P_{i} \times T_{i}$, each $U_{i}$ splits into plaques $\varphi_{i}^{-1}\left(P_{i} \times\{y\}\right)$. Each point $y \in T_{i}$ can also be identified with the point $\varphi_{i}^{-1}(0, y)$ in the local transversal $\varphi_{i}^{-1}\left(\{0\} \times T_{i}\right)$. In addition, the change of charts $\varphi_{j} \circ \varphi_{i}^{-1}: \varphi_{i}\left(U_{i} \cap U_{j}\right) \rightarrow \varphi_{j}\left(U_{i} \cap U_{j}\right)$ is given by

$$
\varphi_{j} \circ \varphi_{i}^{-1}(x, y)=\left(\varphi_{i j}^{y}(x), \gamma_{i j}(y)\right),
$$

where $\gamma_{i j}$ is a homeomorphism between open subsets of $T_{i}$ and $T_{j}$, and $\varphi_{i j}^{y}$ is a $C^{r}$-diffeomorphism depending continuously on $y$ in the $C^{r}$-topology. We say that $\mathscr{A}=\left\{\left(U_{i}, \varphi_{i}\right)\right\}_{i \in I}$ is a good foliated atlas if it satisfies the following conditions.

(i) The cover $\mathcal{U}=\left\{U_{i}\right\}_{i \in I}$ is locally finite, hence finite.

(ii) Each open set $U_{i}$ is a relatively compact subset of a foliated chart.

(iii) If $U_{i} \cap U_{j} \neq \varnothing$, there is a foliated chart containing $\overline{U_{i} \cap U_{j}}$, implying that each plaque of $U_{i}$ intersects at most one plaque of $U_{j}$.

Each foliated chart $U_{i}$ admits a tangentially $C^{r}$-smooth Riemannian metric $g_{i}=$ $\varphi_{i}^{*} g_{0}$ induced from a $C^{r}$-smooth Riemannian metric $g_{0}$ on $\mathbb{R}^{p}$. We can glue together these local Riemannian metrics $g_{i}$ to obtain a global one $g$ using a tangentially $C^{r}$-smooth partition of unity. From [Alcalde Cuesta et al. 2009, Lemma 2.6], 
we know that any $C^{r}$ lamination of a compact space equipped with a $C^{r}$ foliated atlas $\mathscr{A}$ admits a $C^{\infty}$ foliated atlas $C^{r}$-equivalent to $\mathscr{A}$.

A discrete equivalence relation $\mathscr{R}$ is defined by $\mathscr{F}$ on the total transversal $T=$ $\sqcup T_{i}$; the equivalence classes are the traces of the leaves on $T$. We can see $\mathscr{R}$ as the orbit equivalence relation defined by the holonomy pseudogroup $\Gamma$ of $\mathscr{F}$, generated by the local diffeomorphisms $\gamma_{i j}$. These homeomorphisms form a finite generating set, which we will denote by $\Gamma^{(1)}$, that defines a graphing of $\mathscr{R}$. This means that each equivalence class $\mathscr{R}[y]$ is the set of vertices of a graph, and there is an edge joining two vertices $z$ and $w$ if there is $\gamma \in \Gamma^{(1)}$ such that $\gamma(z)=w$. We can define a graph metric $d_{\Gamma}(z, w)=\min \left\{n: g(z)=w\right.$ for some $\left.\gamma \in \Gamma^{(n)}\right\}$, where $\Gamma^{(n)}$ are the elements that can be expressed as words of length at most $n$ in terms of $\Gamma^{(1)}$. A transverse invariant measure for $\mathscr{F}$ is a measure on $T$ that is invariant under the action of $\Gamma$. It is quite rare for a measure of this kind to exist.

Remark 2.1. If $\mathscr{F}$ has no holonomy (that is, $\Gamma_{y}=\{\gamma \in \Gamma: \gamma(y)=y\}$ is trivial for all $y \in T$ ), we can endow $\mathscr{R}$ with the topology generated by the graphs of the elements of $\Gamma$. Then $\mathscr{R}$ becomes an étale equivalence relation, that is, the partial multiplication $\left((y, \gamma(y)),\left(\gamma(y), \gamma^{\prime}(\gamma(y))\right)\right) \in \mathscr{R} * \mathscr{R} \mapsto\left(y, \gamma^{\prime} \circ \gamma(y)\right) \in \mathscr{R}$ and the inversion $(y, \gamma(y)) \in \mathscr{R} \mapsto(\gamma(y), y) \in \mathscr{R}$ are continuous, and the left and right projections $\beta:(y, z) \in \mathscr{R} \mapsto y \in T$ and $\alpha:(y, z) \in \mathscr{R} \mapsto z \in T$ are local homeomorphisms. In general, by considering the germs of the elements of $\Gamma$ at the points of their domains, we can replace $\mathscr{R}$ with the transverse holonomy groupoid [Haefliger 1984] that similarly becomes an étale groupoid [Renault 1980].

2B. Compactly generated pseudogroups. In the last section, we obtained a pseudogroup from a foliated atlas. Here we will recall the Haefliger equivalence for pseudogroups obtained from different atlases and its metric counterpart in the compact case, which we will need later in Section 2C. For any compact laminated space $(M, \mathscr{F})$ the holonomy pseudogroup $\Gamma$ is compactly generated in the sense of [Haefliger 2002], meaning that

(i) $T$ contains a relatively compact open set $T_{1}$ meeting all the orbits, and

(ii) the reduced pseudogroup $\left.\Gamma\right|_{T_{1}}$ (whose elements have domain and range in $T_{1}$ ) admits a finite generating set $\Gamma^{(1)}$ (called a compact generation system of $\Gamma$ on $T_{1}$ ) so that each element $\gamma: A \rightarrow B$ of $\Gamma^{(1)}$ is the restriction of an element $\bar{\gamma}$ of $\Gamma$ whose domain contains the closure of $A$.

Any probability measure $v_{K}$ on the compact set $K=\bar{T}_{1}$ that is preserved by the action of $\left.\Gamma\right|_{K}$ extends to a unique Borel measure $v$ on $T$ which is $\Gamma$-invariant and finite on compact sets. We refer to [Plante 1975, Lemma 3.2].

Also, notice that $T$ is covered by the domains of a family of elements of $\Gamma$ with range in $T_{1}$. The union of these elements and their inverses defines the fundamental 
equivalence between the holonomy pseudogroup $\Gamma$ and the reduced pseudogroup $\left.\Gamma\right|_{T_{1}}$. This is the base concept to define the Haefliger equivalence of pseudogroups [Haefliger 1984; 2002].

Definition 2.2. Two pseudogroups $\Gamma_{1}$ and $\Gamma_{2}$ acting on the spaces $T_{1}$ and $T_{2}$ are Haefliger equivalent if they are reductions of a same pseudogroup $\Gamma$ acting on the disjoint union $T=T_{1} \sqcup T_{2}$, and both $T_{1}$ and $T_{2}$ meet all the orbits of $\Gamma$.

The choice of generators for $\Gamma_{1}$ and $\Gamma_{2}$ defines a metric graph structure on the orbits, but the Haefliger equivalence between $\Gamma_{1}$ and $\Gamma_{2}$ may not preserve their quasi-isometry type. Let us recall this concept introduced by M. Gromov [1993]:

Definition 2.3. Two metric spaces $(M, d)$ and $\left(M^{\prime}, d^{\prime}\right)$ are quasi-isometric if there exists a map $f: M \rightarrow M^{\prime}$ and constants $\lambda \geq 1$ and $C \geq 0$ such that

$$
\frac{1}{\lambda} d(y, z)-C \leq d^{\prime}(f(y), f(z)) \leq \lambda d(y, z)+C
$$

for all $y, z \in M$ and $d^{\prime}\left(y^{\prime}, f(M)\right) \leq C$ for all $y^{\prime} \in M^{\prime}$.

Definition 2.4 [Hurder and Katok 1987; Ghys 1995]. A Haefliger equivalence between two pseudogroups $\Gamma_{1}$ and $\Gamma_{2}$ acting on $T_{1}$ and $T_{2}$, respectively, is a Kakutani equivalence if $\Gamma_{1}$ and $\Gamma_{2}$ admit finite generating systems such that their orbits, endowed with the graph metric, are quasi-isometric.

According to [Lozano Rojo 2006, Theorem 2.7] and [Álvarez López and Candel 2009, Theorem 4.6], if two compactly generated pseudogroups $\Gamma_{1}$ and $\Gamma_{2}$ are Haefliger equivalent, then there are compact generating systems on $T_{1}$ and $T_{2}$, respectively, such that the pseudogroups become Kakutani equivalent. These compact generating systems are called good by Lozano Rojo and recurrent by Álvarez López and Candel. The relevance of this is that the existence of averaging sequences depends on the quasi-isometric type of the orbits; see [Álvarez López and Candel 2009] and [Kanai 1985] for the details.

2C. Existence of transverse invariant measures. In this section we will discuss a sufficient condition for the existence of a transverse invariant measure, which serves as motivation for Theorems 3.6 and 4.10. Goodman and Plante [1979] formulate the following proposition. Let us start with some definitions.

Definition 2.5. Let $A$ be a finite subset of $T$ and $\gamma$ an element of $\Gamma$. We define the difference set

$$
\Delta_{\gamma} A=\{x \in T: x \in A, \gamma(x) \notin A\} \cup\{x \in T: x \notin A, \gamma(x) \in A\},
$$

with the convention that $\gamma(x) \notin A$ holds if $\gamma(x)$ is not defined. We denote the cardinality of $A$ by $|A|$. 
Definition 2.6. A sequence of finite subsets $A_{n}$ of $T$ is an averaging sequence for $\Gamma$ if for all $\gamma \in \Gamma^{(1)}$ (and then for all $\gamma \in \Gamma$ ),

$$
\lim _{n \rightarrow \infty} \frac{\left|\Delta_{\gamma} A_{n}\right|}{\left|A_{n}\right|}=0
$$

Proposition 2.7 [Goodman and Plante 1979]. An averaging sequence $\left\{A_{n}\right\}$ gives rise to a transverse invariant measure $v$ whose support is contained in the limit set $\lim _{n \rightarrow \infty} A_{n}=\left\{y \in T: \exists y_{n_{k}} \in A_{n_{k}}, y=\lim _{k \rightarrow \infty} y_{n_{k}}\right\}$.

The idea of the proof is the following. Assuming that $T$ is compact, we may construct a $\Gamma$-invariant probability measure on $T$ from the sequence of probability measures $v_{n}$ defined by $v_{n}(B)=\left|B \cap A_{n}\right| /\left|A_{n}\right|$ for every Borel set $B \subset T$. By Riesz's representation theorem, each measure $v_{n}$ can be identified with a functional $I_{n}$ on the space $C(T)$ of continuous real-valued functions on $T$. The functionals $I_{n}$ are

$$
I_{n}(f)=\frac{1}{\left|A_{n}\right|} \sum_{y \in A_{n}} f(y) .
$$

By passing to a subsequence, if necessary, $I_{n}$ converges in the weak topology to a positive functional $I$ which determines a unique Borel regular measure $v$ such that $I(f)=\int_{T} f d v$ for every $f \in C(T)$. The averaging condition implies that $I$ and $v$ are $\Gamma$-invariant, since for every $\gamma \in \Gamma$ and every $f \in C(T)$ with support on the range of $\gamma$, we have

$$
|I(f \circ \gamma)-I(f)| \leq\|f\|_{\infty} \lim _{n \rightarrow \infty} \frac{\left|\Delta_{\gamma} A_{n}\right|}{\left|A_{n}\right|}=0 .
$$

Finally, it is clear that $v(T)=1$ and $\operatorname{supp}(v)=\lim _{n \rightarrow \infty} A_{n}$.

In the noncompact case, by replacing $\Gamma$ and $\Gamma_{1}$ with suitable reductions, we can assume, without loss of generality, that the fundamental equivalence between the holonomy pseudogroup $\Gamma$ and its reduction $\Gamma_{1}$ to a relatively compact open subset $T_{1}$ of $T$ becomes a Kakutani equivalence for some compact generation systems on $T$ and $T_{1}$. Then any averaging sequence $A_{n}$ for $\Gamma$ defines an averaging sequence $A_{n} \cap K$ for $\left.\Gamma\right|_{K}$, where $K=\bar{T}_{1}$ is a compact subset of $T$. Hence we obtain a probability measure $v_{K}$ on $K$ that is invariant under $\left.\Gamma\right|_{K}$. Now we can extend $v_{K}$ to a unique Borel measure $v$ on $T$ which is $\Gamma$-invariant and finite on compact sets.

Example 2.8. Consider a graph with bounded geometry, like any orbit $\Gamma(x)$ of the holonomy pseudogroup of a compact laminated space. This graph is said to be Følner if it contains a sequence of finite subsets of vertices $A_{n}$ such that $\left|\partial A_{n}\right| /\left|A_{n}\right|$ tends to 0 , where $\partial A_{n}$ denotes the boundary set with respect to the graph structure. Since $\Delta_{\gamma} A \subset \partial A \cup \gamma^{-1}(\partial A)$ for any $\gamma \in \Gamma^{(1)}$, we get $\left|\Delta_{\gamma} A_{n}\right| \leq 2\left|\partial A_{n}\right|$, and we have an averaging sequence. In particular, any orbit $\Gamma(x)$ having subexponential 
growth is an example of a Følner graph, since

$$
\liminf _{n \rightarrow \infty} \frac{\left|A_{n+1}-A_{n-1}\right|}{\left|A_{n}\right|}=0
$$

where $A_{n}=\Gamma^{(n)}(x)$.

Using the one-to-one correspondence between foliated cycles and transverse invariant measures established by D. Sullivan [1976], it is not difficult to show the following continuous version of Goodman and Plante's result:

Proposition 2.9 [Goodman and Plante 1979]. Let $\left\{V_{n}\right\}$ be an averaging sequence for $\mathscr{F}$, that is, a sequence of compact domains $V_{n}$ (of dimension $d$ ) in the leaves such that

$$
\lim _{n \rightarrow \infty} \frac{\operatorname{area}\left(\partial V_{n}\right)}{\operatorname{vol}\left(V_{n}\right)}=0
$$

where area denotes the $(d-1)$-volume and vol the $d$-volume with respect to the complete Riemannian metric along the leaves. Then $\left\{V_{n}\right\}$ gives rise to a transverse invariant measure $v$ whose support is contained in the saturated limit set $\lim _{n \rightarrow \infty} V_{n}=\left\{p \in M: \exists p_{n_{k}} \in V_{n_{k}}: p=\lim _{k \rightarrow \infty} p_{n_{k}}\right\}$.

Recall that a foliated $d$-form $\alpha \in \Omega^{d}(\mathscr{F})$ is a family of differentiable $d$-forms over the plaques of $\mathscr{A}$ depending continuously on the transverse parameter and agreeing on the intersection of each pair of foliated charts. A foliated $r$-cycle is a continuous linear functional $\xi: \Omega^{d}(\mathscr{F}) \rightarrow \mathbb{R}$ strictly positive on strictly positive forms, and null on exact forms with respect to the leafwise exterior derivative $d \mathscr{F}$. Any averaging sequence $V_{n}$ defines the sequence of foliated currents

$$
\xi_{n}(\alpha)=\frac{1}{\operatorname{vol}\left(V_{n}\right)} \int_{V_{n}} \alpha
$$

where $\alpha$ is a foliated $d$-form. By passing to a subsequence, if necessary, we have a limit current $\xi=\lim _{n \rightarrow \infty} \xi_{n}$. Since the boundaries of the domains $V_{n}$ vanish asymptotically, Stokes' theorem implies that $\xi$ is a foliated $d$-cycle [Sullivan 1976].

\section{Averaging sequences in the discrete setting}

The main objective of this section is to prove the existence of a harmonic measure for an étale equivalence relation $\mathscr{R}$ that contains a modified averaging sequence. Initially, we will assume that $\mathscr{R}$ is given by a free action of a pseudogroup $\Gamma$ on a compact space $T$, but some generalizations will be discussed later. In Section $3 \mathrm{~A}$, we will define a weighted measure on the equivalence classes that will allow us to recall the notion of a modified averaging sequence introduced by V. A. Kaimanovich $[1997 ; 2001]$. Given a continuous cocycle $\delta: \mathscr{R} \rightarrow \mathbb{R}_{+}^{*}$, the Radon-Nikodým problem is to determine the set of probability measures $v$ on $T$ 
which are quasi-invariant and admit $\delta$ as their Radon-Nikodým derivative [Renault 2005]. Theorem 3.6 gives a positive answer to this problem in the presence of a modified averaging sequence.

3A. Quasi-invariant measures. Let $v$ be a quasi-invariant measure on $T$. As usual, we will assume that $v$ is a regular Borel measure that is finite on compact sets. Integrating the counting measures on the fibers of the left projection $\beta(y, z)=y$ with respect to $v$ gives the left counting measure $d \tilde{v}(y, z)=d v(y)$. Indeed, for each Borel set $A \subset \mathscr{R}$, we define

$$
\tilde{v}(A)=\int\left|A^{y}\right| d \mu(y),
$$

where $\left|A^{y}\right|$ is the cardinal of the set $A^{y}=\{z \in T:(y, z) \in A\} \subset \mathscr{R}[y]$. The same is valid for the right projection $\alpha(y, z)=z$, and we get the right counting measure $d \tilde{v}^{-1}(y, z)=d \tilde{v}(z, y)=d v(z)$. Then $\tilde{v}$ and $\tilde{v}^{-1}$ are equivalent measures if and only if $v$ is quasi-invariant, in which case the Radon-Nikodým derivative is given by $\delta(y, z)=d \tilde{v} / d \tilde{v}^{-1}(y, z)$. We refer to [Moore and Schochet 2006; Kaimanovich 1997; Renault 1980; 2005].

Definition 3.1. A cocycle with values in $\mathbb{R}_{+}^{*}$ is a map $\delta: \mathscr{R} \rightarrow \mathbb{R}_{+}^{*}$ satisfying $\delta(x, y) \delta(y, z)=\delta(x, z)$ for all $(x, y),(y, z) \in \mathscr{R}$.

The map $\delta$ is known as the Radon-Nikodým cocycle of $(\mathscr{R}, T, v)$.

Definition 3.2. Given a cocycle $\delta: \mathscr{R} \rightarrow \mathbb{R}_{+}^{*}$, the measure $|\cdot|_{y}$ on $\mathscr{R}[y]$ is given by $|z|_{y}=\delta(z, y)$ for all $z \in \mathscr{R}[y]$. Then, for a finite subset $A \subset \mathscr{R}[y]$,

$$
|A|_{y}=\sum_{z \in A} \delta(z, y) .
$$

3B. Discrete averaging sequences. We want to give a sufficient condition to solve the Radon-Nikodým problem in the discrete setting. We state this condition using the notion of a modified averaging sequence; see [Kaimanovich 1997; 2001]:

Definition 3.3. Let $\delta: \mathscr{R} \rightarrow \mathbb{R}_{+}^{*}$ be a cocycle of $\mathscr{R}$. Let $\left\{A_{n}\right\}$ be a sequence of finite subsets of $T$ such that $A_{n} \subset \mathscr{R}\left[y_{n}\right]$ for each $n \in \mathbb{N}$. We will say that $\left\{A_{n}\right\}$ is a $\delta$-averaging sequence for $\Gamma$ if

$$
\lim _{n \rightarrow \infty} \frac{\left|\Delta_{\gamma} A_{n}\right|_{y_{n}}}{\left|A_{n}\right|_{y_{n}}}=0
$$

for all $\gamma \in \Gamma^{(1)}$. An equivalence class $\mathscr{R}[y]$ is $\delta$-Følner if $\mathscr{R}[y]$ contains a $\delta$ averaging sequence $\left\{A_{n}\right\}$ such that $\left|\partial A_{n}\right|_{y} /\left|A_{n}\right|_{y} \rightarrow 0$ as $n \rightarrow+\infty$.

By choosing a finite generating set for $\Gamma$, we can realize each equivalence class $\mathscr{R}[y]$ as the set of vertices of a graph. We will write $z \sim w$ for each pair of neighboring vertices $z$ and $w$ joined by an edge in $\mathscr{R}[y]$, and $\operatorname{deg} z$ for the number 
of neighbors of $z \in \mathscr{R}[y]$. We will use $\mathscr{D}$ to denote the set of discontinuities of the degree function deg. Let $v$ be a quasi-invariant measure on $T$, and denote by $D: L^{\infty}(T, v) \rightarrow L^{\infty}(T, v)$ the Markov operator defined by

$$
D f(y)=\frac{1}{\operatorname{deg} y} \sum_{z \sim y} f(z) .
$$

We use $D^{*}$ to denote the dual operator acting on the space of positive Borel measures on $T$, and

$$
\Delta: L^{\infty}(T, v) \rightarrow L^{\infty}(T, v)
$$

to denote the Laplace operator defined by $\Delta f(y)=D f(y)-f(y)$.

Definition 3.4. A quasi-invariant measure $v$ on $T$ is harmonic or stationary (for the simple random walk on $\mathscr{R}$ ) if for every bounded measurable function $f: T \rightarrow \mathbb{R}$, we have $\int \Delta f d v=0$.

Proposition 3.5 [Paulin 1999]. For a quasi-invariant measure $v$ on $T$, the following are equivalent:

(i) $v$ is harmonic.

(ii) $D^{*} v=v$.

(iii) The Radon-Nikodým cocycle $\delta: \mathscr{R} \rightarrow \mathbb{R}_{+}^{*}$ is harmonic, that is, for $v$-almost every $y \in T$ and every $z \in \mathscr{R}[y]$, we have

$$
\delta(z, y)=\frac{1}{\operatorname{deg} z} \sum_{w \sim z} \delta(w, y) .
$$

Theorem 3.6. Let $\Re$ be the orbit equivalence relation defined by a finitely generated pseudogroup $\Gamma$ acting freely on a compact space $T$. Let $\delta: \mathscr{R} \rightarrow \mathbb{R}_{+}^{*}$ be a continuous cocycle.

(i) Any $\delta$-averaging sequence $\left\{A_{n}\right\}$ gives rise to a positive Borel measure $v$ on $T$ whose support is contained in the limit set of $\left\{A_{n}\right\}$, which is quasi-invariant and has $\delta$ as its Radon-Nikodým cocycle.

(ii) If $\delta$ is harmonic and $v(D)=0$, then $v$ is a harmonic measure.

Proof. We start by constructing a sequence of probability measures $v_{n}$ given by

$$
v_{n}(B)=\frac{\left|B \cap A_{n}\right|_{y_{n}}}{\left|A_{n}\right|_{y_{n}}}
$$

for every Borel subset $B$ of $T$. By passing to a subsequence, the sequence $v_{n}$ converges in the weak topology to a positive Borel measure $v$ on $T$. First we will prove that $v$ is a quasi-invariant measure having a Radon-Nikodým cocycle equal to $\delta$. For every local transformation $\gamma \in \Gamma$ and every function $f \in C(T)$ with support on the range of $\gamma$, we have 


$$
\int f(z) d\left(\gamma_{*} \nu\right)(z)=\int f(\gamma(y)) d \nu(y)=\lim _{n \rightarrow \infty} \frac{1}{\left|A_{n}\right|_{y_{n}}} \sum_{y \in A_{n}} f(\gamma(y)) \delta\left(y, y_{n}\right)
$$

and

$$
\begin{aligned}
\int f(y) \delta(z, y) d v(y) & =\lim _{n \rightarrow \infty} \frac{1}{\left|A_{n}\right|_{y_{n}}} \sum_{y \in A_{n}} f(y) \delta(\gamma(y), y) \delta\left(y, y_{n}\right) \\
& =\lim _{n \rightarrow \infty} \frac{1}{\left|A_{n}\right|_{y_{n}}} \sum_{y \in A_{n}} f(y) \delta\left(\gamma(y), y_{n}\right),
\end{aligned}
$$

where $z=\gamma(y)$. Therefore

$$
\begin{aligned}
0 & \leq\left|\int f(z) d\left(\gamma_{*} \nu\right)(z)-\int f(y) \delta(z, y) d \nu(y)\right| \\
& \leq \lim _{n \rightarrow \infty} \frac{1}{\left|A_{n}\right|_{y_{n}}}\left|\sum_{y \in A_{n}} f(\gamma(y)) \delta\left(y, y_{n}\right)-f(y) \delta\left(\gamma(y), y_{n}\right)\right| \\
& \leq \lim _{n \rightarrow \infty}\|f\|_{\infty} \frac{\left|\Delta_{\gamma} A_{n}\right|_{y_{n}}}{\left|A_{n}\right|_{y_{n}}}=0,
\end{aligned}
$$

and thus

$$
\int f(z) d\left(\gamma_{*} v\right)(z)=\int f(y) \delta(z, y) d v(y),
$$

proving (i).

We now prove that if $\delta$ is harmonic and $v(\mathscr{D})=0$, then $v$ is a harmonic measure. Observe that if $v(\mathscr{D})=0$, then $\Delta f$ is continuous $v$-almost everywhere, and therefore

$$
\int \Delta f d v=\lim _{n \rightarrow \infty} \int \Delta f d v_{n}
$$

for all $f \in C(T)$. If $\delta$ is harmonic, we have

$$
\begin{aligned}
\int \Delta f(y) d v_{n}(y) & \\
= & \frac{1}{\left|A_{n}\right|_{y_{n}}} \sum_{y \in A_{n}}\left(\frac{1}{\operatorname{deg} y} \sum_{z \sim y} f(z)-f(y)\right) \delta\left(y, y_{n}\right) \\
= & \frac{1}{\left|A_{n}\right|_{y_{n}}} \sum_{y \in A_{n}} \frac{1}{\operatorname{deg} y} \sum_{z \sim y} f(z) \delta\left(y, y_{n}\right)-f(y)\left(\frac{1}{\operatorname{deg} y} \sum_{z \sim y} \delta\left(z, y_{n}\right)\right) \\
= & \frac{1}{\left|A_{n}\right|_{y_{n}}} \sum_{y \in A_{n}} \frac{1}{\operatorname{deg} y} \sum_{z \sim y} f(z) \delta\left(y, y_{n}\right)-f(y) \delta\left(z, y_{n}\right)
\end{aligned}
$$


and then

$$
\begin{aligned}
0 & \leq\left|\int \Delta f(y) d v(y)\right| \\
& \leq \lim _{n \rightarrow \infty} \frac{1}{\left|A_{n}\right|_{y_{n}}}\left|\sum_{y \in A_{n}} \sum_{z \sim y} f(z) \delta\left(y, y_{n}\right)-f(y) \delta\left(z, y_{n}\right)\right| \\
& \leq \lim _{n \rightarrow \infty}\|f\|_{\infty} \sum_{\gamma \in \Gamma^{(1)}} \frac{\left|\Delta_{\gamma} A_{n}\right|_{y_{n}}}{\left|A_{n}\right|_{y_{n}}} \leq \lim _{n \rightarrow \infty} 2\|f\|_{\infty}\left|\Gamma^{(1)}\right| \frac{\left|\partial A_{n}\right|_{y_{n}}}{\left|A_{n}\right|_{y_{n}}}=0 ;
\end{aligned}
$$

that is, $v$ is a harmonic measure.

A similar result can be found in [Schapira 2003]. In general, the second part of Theorem 3.6 remains valid when the Laplace operator $\Delta$ preserves continuous functions. This is always true when $\mathscr{D}=\varnothing$, as in the following case:

Corollary 3.7. Let $\mathscr{R}$ be the orbit equivalence relation defined by a group of finite type $\Gamma$ acting freely on a compact space $T$. Let $\delta: \mathscr{R} \rightarrow \mathbb{R}_{+}^{*}$ be a continuous harmonic cocycle. Any $\delta$-averaging sequence $\left\{A_{n}\right\}$ gives rise to a harmonic measure $v$ on $T$ supported by the limit set of $\left\{A_{n}\right\}$.

Arguing as for usual averaging sequences, we can extend Theorem 3.6 to any compactly generated pseudogroup $\Gamma$ acting freely on a locally compact Polish space $T$. Moreover, in the 0 -dimensional case, the degree function is again continuous. This applies in particular to solenoids [Benedetti and Gambaudo 2003] and laminations defined by repetitive graphs, which were introduced in [Ghys 1999] and studied in [Alcalde Cuesta et al. 2009; Blanc 2001; Lozano Rojo 2011]:

Corollary 3.8. Let $\Re$ be the orbit equivalence relation defined by a compactly generated pseudogroup $\Gamma$ acting freely on a locally compact separable 0-dimensional space $T$. Let $\delta: \mathscr{R} \rightarrow \mathbb{R}_{+}^{*}$ be a continuous harmonic cocycle. Any $\delta$-averaging sequence $\left\{A_{n}\right\}$ gives rise to a harmonic measure $v$ on $T$ supported by the limit set of $\left\{A_{n}\right\}$.

In order to extend Theorem 3.6 to non-free actions, we can adopt two different strategies. Let us first recall that the notion of an equivalence relation is enough to describe the transverse structure of a lamination in the Borel context. More precisely, any Borel or topological lamination $\mathscr{F}$ induces a Borel equivalence relation $\mathscr{R}$ on a total transversal $T$ (compare to Remark 2.1) defined by the action of the holonomy pseudogroup. We refer to the Ph.D. thesis of M. Bermúdez [2004] for the definition of a Borel lamination. If $\mathscr{R}$ is a discrete Borel equivalence relation defined by the action of a Borel pseudogroup $\Gamma$ acting on a compact space $T$ and $\delta: \mathscr{R} \rightarrow \mathbb{R}_{+}^{*}$ is a Borel cocycle, then the proof of Theorem 3.6 remains valid. In the topological context, Theorem 3.6 is not exactly equivalent to the situation above because the transverse holonomy groupoid and the equivalence relation are 
only Borel isomorphic on the residual set of leaves without holonomy. Another strategy consists of replacing étale equivalence relations with étale groupoids and proving that averaging sequences for stationary cocycles define stationary measures on groupoids. Details will be reported elsewhere.

\section{Averaging sequences in the continuous setting}

We are interested in stating Theorem 3.6 in the continuous setting, namely for a compact laminated space $(M, \mathscr{F})$. Instead of working with quasi-invariant measures, we are going to use tangentially smooth measures. These form a larger class than harmonic measures. As previously mentioned, transverse invariant measures for foliations are rather rare, but harmonic measures always exist. Harmonic measures were introduced by L. Garnett [1983]. In Sections 4A and 4B we will study these measures and recall some notation. In Section 4C we will construct a differential foliated 1-form from a given cocycle. Finally, in Section 4D we will use this foliated form to prove the continuous analogue of Theorem 3.6.

4A. Tangentially smooth measures. Consider a regular Borel measure $\mu$ on $M$. Using a $C^{r}$ foliated atlas $\mathscr{A}$, we can give a local decomposition $\mu=\int \lambda_{i}^{y} d \nu_{i}(y)$ on each foliated chart $U_{i}$, where $\lambda_{i}^{y}$ is a measure on the plaque $\varphi_{i}^{-1}\left(P_{i} \times\{y\}\right)$ and $v_{i}$ a measure on $T_{i}$. In order to define the foliated Laplace operator $\Delta_{\mathscr{F}}$, we can always assume that $r \geq 3$ up to $C^{1}$-equivalence of foliated atlases, and we fix a tangentially $C^{r}$-smooth Riemannian metric $g$ along the leaves of $\mathscr{F}$.

Definition 4.1 [Alcalde Cuesta and Rechtman 2011]. A measure $\mu$ on $M$ is tangentially smooth if for every $i \in I$ and $v_{i}$-almost every $y \in T_{i}$, the measures $\lambda_{i}^{y}$ are absolutely continuous with respect to the Riemannian volume $d$ vol restricted to the plaque passing through $y$, and the density functions $h_{i}(x, y)=d \lambda_{i}^{y} / d \operatorname{vol}(x, y)$ are smooth functions of class $C^{r-1}$ on the plaques.

Observe that the local decomposition of $\mu$ is not necessarily unique. Let

$$
\left.\mu\right|_{U_{i}}=\int \lambda_{i}^{y} d v_{i}(y)=\int \bar{\lambda}_{i}^{y} d \bar{\nu}_{i}(y)
$$

be two decompositions. Then we obtain

$$
\int_{T_{i}} \int_{P_{i} \times\{y\}} h_{i}(x, y) d \operatorname{vol}(x, y) d v_{i}(y)=\int_{T_{i}} \int_{P_{i} \times\{y\}} \bar{h}_{i}(x, y) d \operatorname{vol}(x, y) d \bar{v}_{i}(y),
$$

and we can consider the Radon-Nikodým derivative $\delta_{i}(y)=d v_{i} / d \bar{v}_{i}(y)$ such that $\bar{h}_{i}(x, y)=\delta_{i}(y) h_{i}(x, y)$. This situation arises naturally in the intersection of two foliated charts $U_{i}$ and $U_{j}$. Indeed, if $U_{i} \cap U_{j} \neq \varnothing$, we have

$$
\left.\mu\right|_{U_{i} \cap U_{j}}=\int \lambda_{i}^{y} d v_{i}(y)=\int \lambda_{j}^{y} d v_{j}(y) .
$$


Thus, as before, we deduce that

$$
\delta_{i j}(y)=\frac{d \nu_{i}}{d\left(\left(\gamma_{j i}\right)_{*} \nu_{j}\right)}(y)=\frac{h_{j}\left(\varphi_{i j}^{y}(x), \gamma_{i j}(y)\right)}{h_{i}(x, y)} .
$$

Then the functions $h_{i}$ satisfy $\log h_{j}-\log h_{i}=\log \delta_{i j}$ on $U_{i} \cap U_{j}$. Since $\delta_{i j}$ is a function on $T_{i}$, we have that $d_{\mathscr{F}} \log h_{i}=d_{\mathscr{F}} \log h_{j}$. Then $\eta=d_{\mathscr{F}} \log h_{i}$ is a welldefined foliated 1-form of class $C^{r-2}$ along the leaves, which makes it possible to estimate the transverse measure distortion under the holonomy.

Definition 4.2. The foliated 1-form $\eta$ is the modular form of $\mu$.

\section{B. Harmonic measures.}

Definition 4.3 [Garnett 1983]. We will say that $\mu$ is harmonic if $\int \Delta_{\mathscr{F}} f d \mu=0$ for every continuous tangentially $C^{r-1}$-smooth function $f: M \rightarrow \mathbb{R}$.

According to [Garnett 1983, Theorem 1], any harmonic measure is an example of a tangentially smooth measure since the densities $h_{i}$ are positive harmonic functions of class $C^{r-1}$ on the plaques. In particular, any transverse invariant measure combined with the Riemannian volume on the leaves gives a harmonic measure which is called completely invariant. A harmonic measure $\mu$ is completely invariant if and only if $\eta=0$; we refer to [Candel 2003, Corollary 5.5]. In the general harmonic case, the following proposition states some properties of the modular form. This proposition is a refined version of [Deroin 2003, Lemma 4.19].

Proposition 4.4 [Deroin 2003]. If $\mu$ is a harmonic measure, then $\eta$ is a bounded foliated 1-form which admits a uniformly tangentially Lipschitz primitive $\log h$ on the residual set of leaves without holonomy.

Proof. Let $\mathscr{A}=\left\{\left(U_{i}, \phi_{i}\right)\right\}_{i \in I}$ be a good $C^{r}$ foliated atlas of $(M, \mathscr{F})$, and $h_{i}$ the local density functions of $\mu$. Let us first observe that since the functions $h_{i}$ coincide on the intersections of the plaques modulo multiplication by a constant, they define a primitive of the induced 1-form on the holonomy covering of each leaf $L$. If $\mathscr{F}$ has no essential holonomy, the functions $\log h_{i}$ can be glued together to obtain a measurable global primitive $\log h$ of $\eta$. In general, the modular form $\eta$ admits a continuous primitive $\log h$ on the residual set of leaves without holonomy. Now let us assume that $\mathscr{A}$ is a refinement of a good atlas $\mathscr{A}^{\prime}=\left\{\left(U_{i}^{\prime}, \phi_{i}^{\prime}\right)\right\}_{i \in I}$, and $h_{i}^{\prime}$ are the corresponding local densities. Thus, every plaque of $U_{i}$ is relatively compact in a plaque of $U_{i}^{\prime}$. In fact, using a vertical reparametrization, we can suppose that $\phi_{i}^{-1}\left(P_{i} \times\{y\}\right) \subset\left(\phi_{i}^{\prime}\right)^{-1}\left(P_{i}^{\prime} \times\{y\}\right)$ for every $y \in T_{i}$. There exists a relatively compact open set $V \subset P_{i}^{\prime}$ such that $\phi_{i}^{-1}\left(P_{i} \times\{y\}\right) \subset\left(\phi_{i}^{\prime}\right)^{-1}(V \times\{y\})$ for every $y \in T_{i}$. Since $h_{i}$ is harmonic, the Harnack inequality implies the existence of a constant $C_{i}>0$ 
such that

$$
\frac{1}{C_{i}} \leq \frac{h_{i}(x, y)}{h_{i}\left(x_{0}, y\right)} \leq C_{i}
$$

for all $x, x_{0} \in P_{i}$ and for all $y \in T_{i}$. Since the atlases $\mathscr{A}$ and $\mathscr{A}^{\prime}$ are finite, the primitive $\log h$ is uniformly Lipschitz in the tangential coordinate $x$.

4C. Modular form associated to a cocycle. We now describe how to construct a modular 1-form $\eta \in \Omega^{1}(\mathscr{F})$ from a Borel or continuous cocycle $\delta: \mathscr{R} \rightarrow \mathbb{R}_{+}^{*}$. For simplicity, $\mathscr{R}$ is endowed here with the natural Borel or topological structure induced by the Borel or topological groupoid structure on the transverse holonomy groupoid $G$ formed by the germs $\langle\gamma\rangle_{y}$ of the elements $\gamma$ of $\Gamma$ at the points $y$ of their domains; see [Moore and Schochet 2006]. The natural projection

$$
(\beta, \alpha):\langle\gamma\rangle_{y} \in G \mapsto(y, \gamma(y)) \in \mathscr{R}
$$

becomes an isomorphism of Borel or topological groupoids in restriction to the residual set of leaves without holonomy. Equivalently, we can consider a Borel or continuous cocycle $\delta: G \rightarrow \mathbb{R}_{+}^{*}$ projectable on $\mathscr{R}$.

We start by considering tangentially $C^{r}$-smooth Borel or continuous functions $c_{k i}: U_{i} \cap U_{k} \rightarrow \mathbb{R}$ given by

$$
c_{k i}\left(\varphi_{k}^{-1}(x, y)\right)=\log \delta_{k i}(y),
$$

where $\delta_{k i}(y)=\delta\left(y, \gamma_{k i}(y)\right)$ for all $(x, y) \in P_{k} \times T_{k}$. By choosing a tangentially $C^{r}$ smooth partition of unity $\left\{\rho_{i}\right\}_{i=1}^{m}$ subordinated to the foliated atlas $\mathscr{A}$, we can glue the functions $c_{k i}$ obtaining tangentially $C^{r}$-smooth Borel or continuous functions $c_{i}: U_{i} \rightarrow \mathbb{R}$ given by

$$
c_{i}=\sum_{k=1}^{m} \rho_{k} c_{k i}
$$

The cocycle condition implies that $c_{i j}=c_{k j}-c_{k i}$, so that

$$
c_{j}-c_{i}=\sum_{k=1}^{m} \rho_{k} c_{k j}-\sum_{k=1}^{m} \rho_{k} c_{k i}=\left(\sum_{k=1}^{m} \rho_{k}\right) c_{i j}=c_{i j} .
$$

Hence, for each $i=1, \ldots, m$, we can define a tangentially $C^{r-1}$-smooth Borel or continuous foliated 1 -form

$$
\eta_{i}=\sum_{k=1}^{m}\left(d_{\mathscr{F}} \rho_{k}\right) c_{k i}
$$

on $U_{i}$. Each local 1-form $\eta_{i}$ is exact:

$$
\eta_{i}=\sum_{k=1}^{m}\left(d_{\mathscr{F}} \rho_{k}\right) c_{k i}=d_{\mathscr{F}} c_{i}=d_{\mathscr{F}} \log h_{i}
$$


where $h_{i}=e^{c_{i}}: U_{i} \rightarrow \mathbb{R}_{+}^{*}$ is a Borel or continuous function of class $C^{r}$ along the leaves.

Proposition 4.5. There is a well defined Borel or continuous closed foliated 1-form $\eta \in \Omega^{1}(\mathscr{F})$ such that $\left.\eta\right|_{U_{i}}=\eta_{i}$.

Proof. For each pair $i, j \in\{1, \ldots, m\}$, we have that

$$
\eta_{j}-\eta_{i}=\sum_{k=1}^{m}\left(d_{\mathscr{F}} \rho_{k}\right) c_{k j}-\sum_{k=1}^{m}\left(d_{\mathscr{F}} \rho_{k}\right) c_{k i}=\left(\sum_{k=1}^{m} d_{\mathscr{F}} \rho_{k}\right) c_{i j}=0
$$

on $U_{i} \cap U_{j}$. So the 1-form $\eta$ is well defined, Borel, or continuous, and closed.

Definition 4.6. The foliated 1-form $\eta$ is the modular form of $\delta$.

Remark 4.7. (i) The modular form $\eta$ depends on the choice of the partition of unity, but its cohomology class does not.

(ii) As for harmonic measures, the modular form $\eta$ of a Borel or continuous cocycle $\delta$ admits a Borel or continuous primitive $\log h$ on the residual set of leaves without holonomy. Thus, assuming that $\mathscr{F}$ has no holonomy (or passing to the holonomy covers of the leaves), we may find a global Borel or continuous primitive on $M$ (respectively, a Borel or continuous primitive on the holonomy groupoid Hol(FF)); see [Alcalde Cuesta and Rechtman 2011].

4D. Continuous averaging sequences. In the present setting, we can reformulate the Radon-Nikodým problem as the problem of determining tangentially smooth measures $\mu$ on $M$ which admit $\eta$ as their modular form. The aim of this section is to establish Theorem 3.6 for laminations. First we need a continuous analog of Definition 3.3. Consider a $d$-dimensional lamination $\mathscr{F}$ of class $C^{r}$ on a compact space $M$, endowed with a tangentially $C^{r}$-smooth Riemannian metric $g$, and a continuous cocycle $\delta: \mathscr{R} \rightarrow \mathbb{R}_{+}^{*}$. The modular form $\eta$ admits a continuous tangentially $C^{r}$-smooth primitive $\log h$ on the residual set of leaves without holonomy. On each leaf $L_{y}$ without holonomy and passing through $y \in T$, we can multiply $g$ by the normalized density function $h / h(y)$ to obtain a modified metric $(h / h(y)) g$.

Definition 4.8. Let $\left\{V_{n}\right\}$ be a sequence of compact domains with boundary contained in a sequence of leaves without holonomy $L_{y_{n}}$. We will say that $\left\{V_{n}\right\}$ is a $\eta$-averaging sequence for $\mathscr{F}$ if

$$
\lim _{n \rightarrow \infty} \frac{\operatorname{area}_{\eta}\left(\partial V_{n}\right)}{\operatorname{vol}_{\eta}\left(V_{n}\right)}=0
$$

where area $_{\eta}$ denotes the $(d-1)$-volume and $\operatorname{vol}_{\eta}$ the $d$-volume with respect to the modified metric along $L_{y_{n}}$. A leaf $L_{y}$ is $\eta$-F $\phi$ lner if it contains an $\eta$-averaging sequence $\left\{V_{n}\right\}$ such that $\operatorname{area}_{\eta}\left(\partial V_{n}\right) / \operatorname{vol}_{\eta}\left(V_{n}\right) \rightarrow 0$ as $n \rightarrow+\infty$. 
Remark 4.9. (i) The isoperimetric ratio $\operatorname{area}_{\eta}\left(\partial V_{n}\right) / \operatorname{vol}_{\eta}\left(V_{n}\right)$ does not depend on the choice of $y$ or $h$ in the second definition. This justifies our notation here, which differs slightly from that used in [Alcalde Cuesta and Rechtman 2011].

(ii) When $\mu$ is a completely invariant harmonic measure, the normalized density function is equal to one, and thus the modified volume and the Riemannian volume coincide. Hence we recover the common definition of an averaging sequence.

(iii) For harmonic measures, Harnack's inequalities (4-2) imply that the modified volume of the plaques and the modified area of their boundaries remain uniformly bounded.

Theorem 4.10. Let $(M, \mathscr{F})$ be a $C^{r}$ lamination of a compact space $M, 1 \leq r \leq$ $\infty$, and let $\mathscr{R}$ be the equivalence relation induced by $\mathscr{F}$ on a total transversal $T$. Consider a continuous cocycle $\delta: \mathscr{R} \rightarrow \mathbb{R}_{+}^{*}$, and let $\eta$ be the modular form of $\delta$. Assume that $\mathscr{F}$ admits a foliated atlas such that the modified volume of the plaques is bounded.

(i) Any $\eta$-averaging sequence $\left\{V_{n}\right\}$ for $\mathscr{F}$ gives rise to a tangentially smooth measure $\mu$ whose support is contained in the limit set of $\left\{V_{n}\right\}$ and whose modular form is equal to $\eta$.

(ii) If $\eta$ has a primitive $\log h$ such that $h$ is a harmonic function, then $\mu$ is a harmonic measure.

Proof. As in the discrete case, we will start by constructing a sequence of foliated $d$-currents

$$
\xi_{n}(\alpha)=\frac{1}{\operatorname{vol}_{\eta}\left(V_{n}\right)} \int_{V_{n}} \frac{h}{h\left(y_{n}\right)} \alpha,
$$

where $\alpha$ is a foliated $d$-form. By passing to a subsequence, the sequence $\xi_{n}$ converges to a foliated $d$-current $\xi$. Let $\mu$ be the measure on $M$ associated with the current $\xi$. For every function $f \in C(T)$, we have $\int f d \mu=\xi(f \omega)$, where $\omega=d$ vol is the volume form along the leaves.

Now, we will prove that $\mu$ is a tangentially smooth measure with modular form $\eta$. Consider a good $C^{r}$ foliated atlas $\mathscr{A}=\left\{\left(U_{i}, \phi_{i}\right)\right\}_{i \in I}$ obtained by refinement from a given good atlas, and whose plaques have bounded modified volume. As we mentioned before, up to $C^{1}$-equivalence, we can now assume that $r \geq 3$. Since the modified volume of the plaques of $\mathscr{A}$ and the modified area of their boundaries remain bounded, the traces $A_{n}=V_{n} \cap T$ of the domains $V_{n}$ on the total transversal $T$ form a $\delta$-averaging sequence, as in Definition 3.3. In fact, since $V_{n}$ is covered by the plaques $P_{y}$ of $\mathscr{A}$ centered at the points $y$ of $A_{n}$, we have

$$
\operatorname{vol}_{\eta}\left(V_{n}\right)=\int_{V_{n}} \omega_{\eta} \leq \sum_{y \in A_{n}} \int_{P_{y}} \omega_{\eta}=\sum_{y \in A_{n}}\left(\int_{P_{y}} \frac{h(x, y)}{h(0, y)} d \operatorname{vol}(x, y)\right) \delta\left(y, y_{n}\right),
$$


where $\omega_{\eta}$ is the modified volume form along the leaves and $h(x, y)$ denotes the density function restricted to a foliated chart $U_{y}$ containing the plaque $P_{y}$. Then there is a constant $C>0$ such that $\operatorname{vol}_{\eta}\left(V_{n}\right) \leq C\left|A_{n}\right|_{y_{n}}$. Actually, we can choose $C>0$ such that $1 / C \leq \operatorname{vol}_{\eta}\left(V_{n}\right) /\left|A_{n}\right|_{y_{n}} \leq C$. Thus, by passing to a subsequence, we may assume that the $\operatorname{ratio} \operatorname{vol}_{\eta}\left(V_{n}\right) /\left|A_{n}\right|_{y_{n}}$ converges to a constant $c>0$. Now, as stated in the proof of Theorem 3.6, we may also assume that the sequence of measures $v_{n}(B)=\left|B \cap A_{n}\right|_{y_{n}} /\left|A_{n}\right|_{y_{n}}$ converge to a quasi-invariant measure $v$ on $T$ whose Radon-Nikodým derivative is equal to $\delta$. Combined with the modified Riemannian volume along the leaves, this transverse measure gives us a tangentially smooth measure $\mu^{\prime}$ on $M$. Thus, for every function $f \in C(M)$ with support in $U_{i}$, we have

$$
\int f d \mu^{\prime}=\int_{T_{i}} \int_{P_{i} \times\{y\}} f(x, y) \frac{h_{i}(x, y)}{h_{i}(0, y)} d \operatorname{vol}(x, y) d v(y) .
$$

Then

$$
\begin{aligned}
\int f d \mu^{\prime} & =\lim _{n \rightarrow+\infty} \frac{1}{\left|A_{n}\right|_{y_{n}}} \sum_{y \in V_{n} \cap T_{i}}\left(\int_{P_{i} \times\{y\}} f(x, y) \frac{h_{i}(x, y)}{h_{i}(0, y)} d \operatorname{vol}(x, y)\right) \delta\left(y, y_{n}\right) \\
& =\lim _{n \rightarrow+\infty} \frac{1}{\left|A_{n}\right|_{y_{n}}} \sum_{y \in V_{n} \cap T_{i}} \int_{P_{i} \times\{y\}} f \omega_{\eta} .
\end{aligned}
$$

On the other hand, by definition, we have

$$
\begin{aligned}
\int f d \mu=\xi(f \omega) & =\lim _{n \rightarrow+\infty} \frac{1}{\operatorname{vol}_{\eta}\left(V_{n}\right)} \int_{V_{n}} f \omega_{\eta} \\
& =\lim _{n \rightarrow+\infty} \frac{1}{\operatorname{vol}_{\eta}\left(V_{n}\right)} \sum_{y \in V_{n} \cap T_{i}} \int_{P_{i} \times\{y\}} f \omega_{\eta} .
\end{aligned}
$$

Comparing identities (4-3) and (4-4), we deduce that $\mu=(1 / c) \mu^{\prime}$ is a tangentially smooth measure with modular form $\eta$.

To conclude, we will prove that $\mu$ is harmonic when $h$ is harmonic. We will start by denoting the normalized density functions on the leaves $L_{y_{n}}$ by $h_{n}=h / h\left(y_{n}\right)$. Since the Laplace operator $\Delta_{\mathscr{F}}$ preserves continuous functions, we have

$$
\int \Delta_{\mathscr{F}} f d \mu=\lim _{n \rightarrow \infty} \frac{1}{\operatorname{vol}_{h}\left(V_{n}\right)} \int_{V_{n}}\left(\Delta_{\mathscr{F}} f\right) h_{n} \omega
$$

for all $f \in C(T)$. Green's formula implies that

$$
\int_{V_{n}}\left(\Delta_{\mathscr{F}} f\right) h_{n} \omega=\int_{V_{n}}\left(\left(\Delta_{\mathscr{F}} f\right) h_{n}-f\left(\Delta_{\mathscr{F}} h_{n}\right) \omega=\int_{\partial V_{n}} h_{n} \iota_{\operatorname{grad}(f)} \omega-f \iota_{\operatorname{grad}\left(h_{n}\right)} \omega .\right.
$$

Since $h_{n}$ is harmonic, we have

$$
\int_{\partial V_{n}} \iota_{\operatorname{grad}\left(h_{n}\right)} \omega=\int_{V_{n}} \operatorname{div}\left(\operatorname{grad}\left(h_{n}\right)\right) \omega=\int_{V_{n}}\left(\Delta_{\mathscr{F}} h_{n}\right) \omega=0
$$


and then

$$
0 \leq\left|\int_{\partial V_{n}} f \iota_{\operatorname{grad}\left(h_{n}\right)} \omega\right| \leq\|f\|_{\infty} \int_{\partial V_{n}} \iota_{\operatorname{grad}\left(h_{n}\right)} \omega=0
$$

for all $n \in \mathbb{N}$. On the other hand, since $f$ is bounded, there exists a constant $k>0$ depending only on $f$, such that

$$
0 \leq\left|\frac{1}{\operatorname{vol}_{h}\left(V_{n}\right)} \int_{\partial V_{n}} h_{n} \iota_{\operatorname{grad}(f)} \omega\right| \leq \lim _{n \rightarrow \infty} k \frac{\operatorname{area}_{\eta}\left(\partial V_{n}\right)}{\operatorname{vol}_{\eta}\left(V_{n}\right)}=0 .
$$

Therefore

$$
\int \Delta_{\mathscr{F}} f d \mu=\lim _{n \rightarrow \infty} \frac{1}{\operatorname{vol}_{h}\left(V_{n}\right)} \int_{V_{n}}\left(\Delta_{\mathscr{F}} f\right) h_{n} \omega=0,
$$

that is, $\mu$ is a harmonic measure.

Remark 4.11. (i) If $\delta: \mathscr{R} \rightarrow \mathbb{R}_{+}^{*}$ is a Borel cocycle with modular form $\eta$, Theorem 4.10 also remains valid. So any $\eta$-averaging sequence for $\mathscr{F}$ gives rise to a tangentially smooth measure $\mu$ that is harmonic when $\eta$ admits a primitive $\log h$ such that $h$ is a harmonic function.

(ii) According to Remark 4.7(ii), the notion of $\eta$-Følner may be applied to the holonomy covers of the leaves of $\mathscr{F}$. Thus it suffices to replace $\mathscr{F}$ with the lifted lamination in the holonomy groupoid $\mathrm{Hol}(\mathscr{F})$ in order to globalize the previous result. As in the discrete setting, details will be discussed elsewhere.

\section{Examples}

5A. Discrete averaging sequences for amenable non-Følner actions. There are amenable actions of nonamenable discrete groups whose orbits contain averaging sequences [Kaimanovich 2001]. For example, let $\partial \Gamma$ be the space of ends of the free group $\Gamma$ with two generators $\alpha$ and $\beta$ whose elements are infinite words $x=\gamma_{1} \gamma_{2} \ldots$ with letters $\gamma_{n}$ in $\Phi=\left\{\alpha^{ \pm 1}, \beta^{ \pm 1}\right\}$. If $v$ denotes the equidistributed probability measure on $\partial \Gamma$ (such that all cylinders consisting of infinite words with fixed first $n$ letters have the same measure), then $\Gamma$ acts essentially freely on $\partial \Gamma$ by sending each generator $\gamma$ and each infinite word $x=\gamma_{1} \gamma_{2} \ldots$ to $\gamma . x=\gamma \gamma_{1} \gamma_{2} \ldots$ Since this action is amenable, according to [Kaimanovich 1997, Theorem 2], we know that $\nu$-almost every orbit is $\delta$-Følner (where $\delta$ is the Radon-Nikodým derivative of $v$ ); see also [Alcalde Cuesta and Rechtman 2011, Proposition 4.1]. We recall here an explicit construction by Kaimanovich [2001].

For each $x \in \partial \Gamma$, let $b_{x}: \Gamma \rightarrow \mathbb{R}$ be the Busemann function defined by

$$
b_{x}(\gamma)=\lim _{n \rightarrow+\infty}\left(d_{\Gamma}\left(\gamma, x_{[n]}\right)-d_{\Gamma}\left(1, x_{[n]}\right)\right),
$$

where $d_{\Gamma}$ is the Cayley graph metric, $x_{[n]}$ is the word consisting of the first $n$ letters of $x$, and 1 is the identity element. The level sets $H_{k}(x)=\left\{\gamma \in \Gamma: b_{x}(\gamma)=k\right\}$, are 
the horospheres centered at $x$. The Radon-Nikodým derivative of $v$ is given by

$$
\delta\left(\gamma^{-1} \cdot x, x\right)=\frac{d \gamma \cdot \nu}{d \nu}(x)=3^{-b_{x}(\gamma)},
$$

where $\gamma \cdot v$ is the translation of $v$ by $\gamma$. Since $|\cdot|_{x}=\delta(\cdot, x)$ is a harmonic measure on $\Gamma . x, v$ is also a harmonic measure. In fact, as stated in [Kaimanovich 2000, Theorem 17.4], $v$ is the unique harmonic probability measure on $\partial \Gamma$.

Let $A_{n}^{x}$ be the set of all points $\gamma^{-1} . x$ in $\Gamma . x$ such that $0 \leq b_{x}(\gamma)=d_{\Gamma}(1, \gamma) \leq n$. Since

$$
\left|A_{n}^{x} \cap H_{k}(x)\right|_{x}=\sum_{b_{x}(\gamma)=d_{\Gamma}(1, \gamma)=k} \delta\left(\gamma^{-1} . x, x\right)=3^{k} \frac{1}{3^{k}}=1
$$

for all $0 \leq k \leq n$, we have that $\left|A_{n}\right|_{x}=n+1$. But $\partial A_{n}^{x}=\{1\} \cup\left(A_{n}^{x} \cap H_{n}(x)\right)$, and so $\left|\partial A_{n}^{x}\right|_{x}=2$. The $\delta$-averaging sequence $\left\{A_{n}^{x}\right\}$ defines a harmonic measure (which is equal to $v$ up to multiplication by a constant).

5B. Averaging sequences for hyperbolic surfaces. The geodesic and horocycle flows are classical examples of flows on the unitary tangent bundle of a compact hyperbolic surface. They are given by the right actions of the diagonal subgroup

$$
D=\left\{\left(\begin{array}{cc}
e^{t / 2} & 0 \\
0 & e^{-t / 2}
\end{array}\right): t \in \mathbb{R}\right\}
$$

and the unipotent subgroup

$$
H^{+}=\left\{\left(\begin{array}{ll}
1 & s \\
0 & 1
\end{array}\right): s \in \mathbb{R}\right\}
$$

of $G=\operatorname{PSL}(2, \mathbb{R})$ on the quotient $\Gamma \backslash G$ by the left action of a uniform lattice $\Gamma$. If $\mathbb{U}$ denotes the hyperbolic plane, we can identify $\Gamma \backslash G$ with the unitary tangent bundle of the compact hyperbolic surface $\Gamma \backslash \mathbb{H}$. The right action of the normalizer $A$ of $H^{+}$in PSL $(2, \mathscr{R})$ defines a foliation $\mathscr{F}$ by Riemann surfaces on $\Gamma \backslash G$. Since $A$ is an amenable group, $\mathscr{F}$ is an amenable non-Følner foliation. Moreover, there is an $A$-invariant measure $\mu$ on $\Gamma \backslash G$. Garnett [1983] proved that $\mu$ is a harmonic measure by describing its density function on a foliated chart.

We can identify $G / A$ with the boundary $\partial \llbracket$ by sending each coset of $A$ in $G$ to the center of the horocycle defined by the corresponding coset of $\mathrm{H}^{+}$in $G$. For each point $z \in \mathbb{M}$, there is a unique probability measure $v_{z}$ on $\partial \mathbb{U}$ which is invariant by the action of all isometries of $\mathbb{H}$ fixing $z$. This measure is the image of the normalized Lebesgue measure on the circle of the tangent plane at $z$ under the exponential map, and is called the visual measure at $z$. According to [Garnett 1983, Proposition 2], the normalized density function is given by $d v_{z} / d v_{z_{0}}(x)$ where 
$z, z_{0} \in \mathbb{U}$ and $x \in \partial \mathbb{H}$. In particular, for $x=\infty$, we have

$$
\frac{d v_{z}}{d v_{z_{0}}}(\infty)=\frac{y}{y_{0}},
$$

where $z=x+i y$ and $z_{0}=x_{0}+i y_{0}$. In the leaf passing through $x=\infty$, the sequence $V_{n}^{\infty}=\left\{z \in \mathbb{H}:-1 \leq x \leq 1, e^{-n} \leq y \leq 1\right\}$ becomes an $\eta$-averaging sequence (where $\eta$ is the modular form of $\mu$ ). Indeed, on the one hand, we have

$$
\operatorname{area}_{\eta}\left(V_{n}^{\infty}\right)=\int_{V_{n}^{\infty}} \frac{d \nu_{z}}{d \nu_{i}}(\infty) d \operatorname{vol}(z)=\int_{V_{n}^{\infty}} y \frac{d x \wedge d y}{y^{2}}=\int_{1}^{1} d x \int_{e^{-n}}^{1} \frac{d y}{y}=2 n .
$$

On the other hand, the modified length of a smooth curve $\sigma(t)=x(t)+i y(t)$ (with $0 \leq t \leq l)$ is given by length ${ }_{\eta}(\sigma)=\int_{0}^{l} \sqrt{x^{\prime}(t)^{2}+y^{\prime}(t)^{2}} d t$, and so we have

$$
\text { length }_{\eta}\left(\partial V_{n}^{\infty}\right)=2\left(2+\left(1-e^{n}\right)\right) \leq 6 .
$$

As before, this $\eta$-averaging sequence defines a harmonic measure (which is equal to $\mu$ up to multiplication by a constant). In fact, all leaves are $\eta$-Følner since for each point $x \in \partial \mathbb{U}$ obtained as the image of $\infty$ under $g \in G$, the sets $V_{n}^{x}=g\left(V_{n}^{\infty}\right)$ form an $\eta$-averaging sequence in the leaf passing through $x$.

5C. Averaging sequences for torus bundles over the circle. In conclusion, we will now present other examples of foliations on homogeneous spaces studied by É. Ghys and V. Sergiescu [1980]. Each matrix $A \in \operatorname{SL}(2, \mathbb{Z})$ with $|\operatorname{tr}(A)|>2$ defines a natural representation $\varphi: \mathbb{Z} \rightarrow \operatorname{Aut}\left(\mathbb{Z}^{2}\right)$ which extends to a representation $\Phi: \mathbb{R} \rightarrow \operatorname{Aut}\left(\mathbb{R}^{2}\right)$ given by $\Phi(t)=A^{t}$. If $\lambda>1$ and $\lambda^{-1}<1$ are the eigenvalues of $A$, then $\Phi$ is conjugated to the representation $\Phi_{0}$ given by

$$
\Phi_{0}(t)=\left(\begin{array}{cc}
\lambda^{t} & 0 \\
0 & \lambda^{-t}
\end{array}\right)
$$

Let $T_{A}^{3}$ be the homogeneous space obtained as the quotient of the Lie group $G=$ $\mathbb{R}^{2} \rtimes_{\Phi} \mathbb{R}$ with group law $(x, y, t) .\left(x^{\prime}, y^{\prime}, t^{\prime}\right)=\left((x, y)+A^{t}\left(x^{\prime}, y^{\prime}\right), t+t^{\prime}\right)$ by the uniform lattice $\Gamma=\mathbb{Z}^{2} \rtimes_{\varphi} \mathbb{Z}$ with a similar law. Observe that $G$ is isomorphic to the solvable group $\mathrm{Sol}^{3}=\mathbb{R}^{2} \rtimes_{\Phi_{0}} \mathbb{R}$ with group law

$$
(x, y, t) \cdot\left(x^{\prime}, y^{\prime}, t^{\prime}\right)=\left(x+\lambda^{t} x^{\prime}, y+\lambda^{-t} y^{\prime}, t+t^{\prime}\right)
$$

(where $x$ and $y$ are the first and second coordinate with respect to the eigenbasis) and $T_{A}^{3}$ is diffeomorphic to the quotient of $\mathrm{Sol}^{3}$ by a uniform lattice $\Gamma_{0}$. The right action of the image $A$ of the monomorphism

$$
(a, b) \in \mathbb{R} \rtimes \mathbb{R}_{+}^{*} \mapsto\left(a, 0, \frac{\log b}{\log \lambda}\right) \in \operatorname{Sol}^{3}
$$

defines a foliation $\mathscr{F}$ on $T_{A}^{3}$. The Lebesgue measure on $T_{A}^{3}$ defined by the volume form $\Omega=d x \wedge d y \wedge d t$ is a tangentially smooth measure. Since the Riemannian 
volume along the right orbits is given by

$$
\frac{d a \wedge d b}{b^{2}}=(\log \lambda) \lambda^{-t} d x \wedge d t,
$$

the density function is equal to $\lambda^{t} / \log \lambda$. In the orbit of the identity element, the sequence $V_{n}=\left\{(a, b) \in A:-1 \leq a \leq 1, e^{-n \log \lambda} \leq b \leq 1\right\}$ becomes an $\eta$-averaging sequence (where $\eta$ is the modular form of $\mu$ ). Indeed, on the one hand, we have

$$
\operatorname{area}_{\eta}\left(V_{n}\right)=\int_{V_{n}} \frac{1}{\log \lambda} \lambda^{t}(\log \lambda) \lambda^{-t} d x \wedge d t=\int_{1}^{1} d x \int_{-n}^{0} d t=2 n .
$$

On the other hand, the modified length of a smooth curve $\sigma(t)=(a(t), b(t))$ (with $0 \leq t \leq L)$ is given by length ${ }_{\eta}(\sigma)=\int_{0}^{L} \sqrt{a^{\prime}(t)^{2}+b^{\prime}(t)^{2}} d t$, and so we have that

$$
\text { length }_{\eta}\left(\partial V_{n}\right)=2\left(2+\left(1-e^{n \log \lambda}\right)\right) \leq 6 .
$$

By replacing the orbit corresponding to $y=0$ with another orbit, it is easy to see that all leaves are $\eta$-Følner. As in the previous example, all $\eta$-averaging sequences define (up to multiplication by a constant) the same harmonic measure, the Lebesgue measure.

\section{Final comments}

6A. Discrete and continuous averaging sequences. Comparing the discrete and continuous settings, a natural question arises: what is the relation between $\delta$ averaging and $\eta$-averaging sequences? Let us first notice that repeating the same argument as in the classical case (see [Kanai 1985, Theorem 4.1]), the boundedness condition derived from Harnack's inequalities in Remark 4.9(iii) implies that the

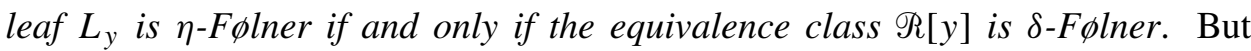
then what is the relation between the harmonic measures defined by $\delta$-averaging and $\eta$-averaging sequences? In this case, the answer is more subtle, and we have to use an important result of R. Lyons and Sullivan [1984], completed later by Kaimanovich [1992] and, independently, by W. Ballman and F. Ledrappier [1996], about the discretization of harmonic functions on Riemannian manifolds. First, according to [Lyons and Sullivan 1984, Theorem 6], if $\mu$ is a harmonic measure, then the transverse measure $v$ (well defined up to equivalence) is $\pi$-harmonic, where $\pi$ is a transition kernel defining a random walk on $\mathscr{R}$, different from the simple random walk considered in Definition 3.4. Reciprocally, assuming that $T$ admits a relatively compact neighborhood which meets almost every leaf in a recurrent set, [Ballmann and Ledrappier 1996, Main Theorem] implies that $\mu$ is harmonic if $v$ is $\pi$-harmonic.

6B. Amenability. It is not a coincidence that all the examples in Section 5 are amenable: according to a result of Kaimanovich [1997], amenable foliations admit 
always averaging sequences. In fact, if $\mathscr{F}$ is an amenable foliation with respect to a tangentially smooth measure $\mu$, then $\mathscr{F}$ is $\eta$ - Følner, that is, $\mu$-almost every leaf is $\eta$-Følner; see [Alcalde Cuesta and Rechtman 2011, Proposition 4.3]. This paper can be viewed as a sequel to [Alcalde Cuesta and Rechtman 2011] where we proved that minimal $\eta$-Følner foliations are $\mu$-amenable (assuming that the modified volume of the plaques is bounded). To complete the series, we have to prove that any foliation is amenable with respect to a tangentially smooth measure $\mu$ constructed from an averaging sequence using Theorem 4.10.

\section{References}

[Alcalde Cuesta and Rechtman 2011] F. Alcalde Cuesta and A. Rechtman, "Minimal Følner foliations are amenable", Discrete Contin. Dyn. Syst. 31:3 (2011), 685-707. MR 2825634 Zbl 05988150

[Alcalde Cuesta et al. 2009] F. Alcalde Cuesta, A. Lozano Rojo, and M. Macho Stadler, "Dynamique transverse de la lamination de Ghys-Kenyon", pp. 1-16 in Équations différentielles et singularités: en l'honneur de J. M. Aroca, edited by F. Cano et al., Astérisque 323, Soc. Math. France, Paris, 2009. MR 2011g:37062 Zbl 1203.37011

[Álvarez López and Candel 2009] J. A. Álvarez López and A. Candel, "Equicontinuous foliated spaces", Math. Z. 263:4 (2009), 725-774. MR 2010i:53040 Zbl 1177.53026

[Ballmann and Ledrappier 1996] W. Ballmann and F. Ledrappier, "Discretization of positive harmonic functions on Riemannian manifolds and Martin boundary", pp. 77-92 in Actes de la Table Ronde de Géométrie Différentielle: en l'honneur de Marcel Berger (Luminy, 1992), edited by A. L. Besse, Sémin. Congr. 1, Soc. Math. France, Paris, 1996. MR 97m:58207 Zbl 0885.53037

[Benedetti and Gambaudo 2003] R. Benedetti and J.-M. Gambaudo, "On the dynamics of $\mathbb{G}$-solenoids: applications to Delone sets”, Ergodic Theory Dynam. Systems 23:3 (2003), 673-691. MR 2004f:37019 Zbl 1124.37009

[Bermúdez 2004] M. Bermúdez, Laminations Boréliennes, thesis, Université Claude Bernard, Lyon, 2004, Available at http://tinyurl.com/Bermudez-2004.

[Blanc 2001] E. Blanc, Propriétés génériques des laminations, thesis, Université Claude Bernard, Lyon, 2001.

[Candel 2003] A. Candel, "The harmonic measures of Lucy Garnett", Adv. Math. 176:2 (2003), 187-247. MR 2004m:58057 Zbl 1031.58003

[Deroin 2003] B. Deroin, Laminations par variétés complexes, thesis, École Normale Supérieure de Lyon, 2003.

[Følner 1955] E. Følner, "On groups with full Banach mean value”, Math. Scand. 3 (1955), 243-254. MR 18,51f Zbl 0067.01203

[Garnett 1983] L. Garnett, "Foliations, the ergodic theorem and Brownian motion", J. Funct. Anal. 51:3 (1983), 285-311. MR 84j:58099 Zbl 0524.58026

[Ghys 1995] É. Ghys, "Topologie des feuilles génériques”, Ann. of Math. (2) 141:2 (1995), 387422. MR 96b:57032 Zbl 0843.57026

[Ghys 1999] É. Ghys, "Laminations par surfaces de Riemann", pp. ix, xi, 49-95 in Dynamique et géométrie complexes (Lyon, 1997), Panor. Synthèses 8, Soc. Math. France, Paris, 1999. In French; translated by L. Kay in Complex dynamics and geometry, SMF/AMS Texts and Monographs 10, Amer. Soc. Math., Providence, RI, 2003, pp. 43-84. MR 2001g:37068 Zbl 1018.37028 
[Ghys and Sergiescu 1980] É. Ghys and V. Sergiescu, "Stabilité et conjugaison différentiable pour certains feuilletages”, Topology 19:2 (1980), 179-197. MR 81k:57022 Zbl 0478.57017

[Goodman and Plante 1979] S. E. Goodman and J. F. Plante, "Holonomy and averaging in foliated sets”, J. Differential Geom. 14:3 (1979), 401-407. MR 81m:57020 Zbl 0475.57007

[Gromov 1993] M. Gromov, "Asymptotic invariants of infinite groups", pp. 1-295 in Geometric group theory (Sussex, 1991), vol. 2, edited by G. A. Niblo and M. A. Roller, London Math. Soc. Lecture Note Ser. 182, Cambridge University Press, Cambridge, 1993. MR 95m:20041 Zbl 0841. 20039

[Haefliger 1984] A. Haefliger, "Groupoïdes d'holonomie et classifiants", pp. 70-97 in Structure transverse des feuilletages (Toulouse, 1982), edited by J. Pradines, Astérisque 116, Soc. Math. France, Paris, 1984. MR 86c:57026a Zbl 0562.57012

[Haefliger 2002] A. Haefliger, "Foliations and compactly generated pseudogroups", pp. 275-295 in Foliations: geometry and dynamics (Warsaw, 2000), edited by P. Walczak et al., World Scientific, River Edge, NJ, 2002. MR 2003g:58029 Zbl 1002.57059

[Hurder and Katok 1987] S. Hurder and A. Katok, "Ergodic theory and Weil measures for foliations”, Ann. of Math. (2) 126:2 (1987), 221-275. MR 89d:57042 Zbl 0645.57021

[Kaimanovich 1992] V. A. Kaimanovich, "Discretization of bounded harmonic functions on Riemannian manifolds and entropy", pp. 213-223 in Potential theory (Nagoya, 1990), edited by M. Kishi, de Gruyter, Berlin, 1992. MR 94b:31007 Zbl 0768.58054

[Kaimanovich 1997] V. A. Kaimanovich, "Amenability, hyperfiniteness, and isoperimetric inequalities”, C. R. Acad. Sci. Paris Sér. I Math. 325:9 (1997), 999-1004. MR 98j:28014 Zbl 0981.28014

[Kaimanovich 2000] V. A. Kaimanovich, "The Poisson formula for groups with hyperbolic properties”, Ann. of Math. (2) 152:3 (2000), 659-692. MR 2002d:60064 Zbl 0984.60088

[Kaimanovich 2001] V. A. Kaimanovich, "Equivalence relations with amenable leaves need not be amenable", pp. 151-166 in Topology, ergodic theory, real algebraic geometry, edited by V. Turaev and A. Vershik, Amer. Math. Soc. Transl. Ser. 2 202, Amer. Math. Soc., Providence, RI, 2001. MR 2003a:37009 Zbl 0990.28013

[Kanai 1985] M. Kanai, "Rough isometries, and combinatorial approximations of geometries of noncompact Riemannian manifolds", J. Math. Soc. Japan 37:3 (1985), 391-413. MR 87d:53082 Zbl 0554.53030

[Lozano Rojo 2006] Á. Lozano Rojo, “The Cayley foliated space of a graphed pseudogroup”, pp. 267-272 in XIV Fall Workshop on Geometry and Physics (Bilbao, 2005), edited by L. C. de Andrés et al., Publ. R. Soc. Mat. Esp. 8, R. Soc. Mat. Esp., Madrid, 2006. MR 2008j:58027 Zbl 1158.58008

[Lozano Rojo 2011] Á. Lozano Rojo, “An example of a non-uniquely ergodic lamination”, Ergodic Theory Dynam. Systems 31:2 (2011), 449-457. MR 2776384 Zbl 1221.37049

[Lyons and Sullivan 1984] T. Lyons and D. Sullivan, "Function theory, random paths and covering spaces”, J. Differential Geom. 19:2 (1984), 299-323. MR 86b:58130 Zbl 0554.58022

[Moore and Schochet 2006] C. C. Moore and C. L. Schochet, Global analysis on foliated spaces, 2nd ed., Mathematical Sciences Research Institute Publications 9, Cambridge University Press, New York, 2006. MR 2006i:58035 Zbl 1091.58015

[Paulin 1999] F. Paulin, "Propriétés asymptotiques des relations d'équivalences mesurées discrètes", Markov Process. Related Fields 5:2 (1999), 163-200. MR 2001m:37010 Zbl 0937.28015

[Plante 1975] J. F. Plante, "Foliations with measure preserving holonomy", Ann. of Math. (2) 102:2 (1975), 327-361. MR 52 \#11947 Zbl 0314.57018

[Renault 1980] J. Renault, A groupoid approach to $C^{*}$-algebras, Lecture Notes in Mathematics 793, Springer, Berlin, 1980. MR 82h:46075 Zbl 0433.46049 
[Renault 2005] J. Renault, "The Radon-Nikodým problem for approximately proper equivalence relations", Ergodic Theory Dynam. Systems 25:5 (2005), 1643-1672. MR 2006h:46065 Zbl 1093. 46035

[Schapira 2003] B. Schapira, "Mesures quasi-invariantes pour un feuilletage et limites de moyennes longitudinales", C. R. Math. Acad. Sci. Paris 336:4 (2003), 349-352. MR 2004j:37043 Zbl 1030. 57043

[Sullivan 1976] D. Sullivan, "Cycles for the dynamical study of foliated manifolds and complex manifolds”, Invent. Math. 36:1 (1976), 225-255. MR 55 \#6440 Zbl 0335.57015

Received January 8, 2011. Revised May 26, 2011.

FERnANdo AlCAlde Cuesta

DEPARTAMENTO DE XeOMETRÍA E TOPOlOXÍA

UNIVERSIDADE DE SANTIAGO DE COMPOSTELA

RÚA LOPE GÓMEZ DE MARZOA S/N

15782 SANTIAGO DE COMPOSTELA

SPAIN

fernando.alcalde@usc.es

ANA RECHTMAN

Institut De Recherche MathématiQue AvancÉE

UNIVERSITÉ DE STRASBOURG

7 RUE RENÉ DESCARTES

67084 STRASBOURG

FRANCE

rechtman@unistra.fr 


\title{
PACIFIC JOURNAL OF MATHEMATICS
}

\author{
http://pacificmath.org \\ Founded in 1951 by \\ E. F. Beckenbach (1906-1982) and F. Wolf (1904-1989)
}

\section{EDITORS}

V. S. Varadarajan (Managing Editor)

Department of Mathematics

University of California

Los Angeles, CA 90095-1555

pacific@math.ucla.edu

Vyjayanthi Chari

Department of Mathematics

University of California

Riverside, CA 92521-0135

chari@math.ucr.edu

\section{Robert Finn}

Department of Mathematics Stanford University

Stanford, CA 94305-2125

finn@math.stanford.edu

Kefeng Liu

Department of Mathematics

University of California

Los Angeles, CA 90095-1555

liu@math.ucla.edu
Darren Long

Department of Mathematics

University of California

Santa Barbara, CA 93106-3080

long@math.ucsb.edu

Jiang-Hua Lu

Department of Mathematics

The University of Hong Kong

Pokfulam Rd., Hong Kong jhlu@maths.hku.hk

Alexander Merkurjev

Department of Mathematics

University of California

Los Angeles, CA 90095-1555

merkurev@math.ucla.edu
Sorin Popa

Department of Mathematics University of California

Los Angeles, CA 90095-1555 popa@math.ucla.edu

Jie Qing

Department of Mathematics

University of California

Santa Cruz, CA 95064

qing@cats.ucsc.edu

Jonathan Rogawski

Department of Mathematics

University of California

Los Angeles, CA 90095-1555

jonr@math.ucla.edu

\section{PRODUCTION}

pacific@math.berkeley.edu

\section{SUPPORTING INSTITUTIONS}

ACADEMIA SINICA, TAIPEI

CALIFORNIA INST. OF TECHNOLOGY INST. DE MATEMÁTICA PURA E APLICADA KEIO UNIVERSITY

MATH. SCIENCES RESEARCH INSTITUTE NEW MEXICO STATE UNIV.

OREGON STATE UNIV.

\author{
STANFORD UNIVERSITY \\ UNIV. OF BRITISH COLUMBIA \\ UNIV. OF CALIFORNIA, BERKELEY \\ UNIV. OF CALIFORNIA, DAVIS \\ UNIV. OF CALIFORNIA, LOS ANGELES \\ UNIV. OF CALIFORNIA, RIVERSIDE \\ UNIV. OF CALIFORNIA, SAN DIEGO \\ UNIV. OF CALIF., SANTA BARBARA
}

\author{
UNIV. OF CALIF., SANTA CRUZ \\ UNIV. OF MONTANA \\ UNIV. OF OREGON \\ UNIV. OF SOUTHERN CALIFORNIA \\ UNIV. OF UTAH \\ UNIV. OF WASHINGTON \\ WASHINGTON STATE UNIVERSITY
}

These supporting institutions contribute to the cost of publication of this Journal, but they are not owners or publishers and have no responsibility for its contents or policies.

See inside back cover or pacificmath.org for submission instructions.

The subscription price for 2012 is US \$420/year for the electronic version, and \$485/year for print and electronic.

Subscriptions, requests for back issues from the last three years and changes of subscribers address should be sent to Pacific Journal of Mathematics, P.O. Box 4163, Berkeley, CA 94704-0163, U.S.A. Prior back issues are obtainable from Periodicals Service Company, 11 Main Street, Germantown, NY 12526-5635. The Pacific Journal of Mathematics is indexed by Mathematical Reviews, Zentralblatt MATH, PASCAL CNRS Index, Referativnyi Zhurnal, Current Mathematical Publications and the Science Citation Index.

The Pacific Journal of Mathematics (ISSN 0030-8730) at the University of California, c/o Department of Mathematics, 969 Evans Hall, Berkeley, CA 94720-3840, is published monthly except July and August. Periodical rate postage paid at Berkeley, CA 94704, and additional mailing offices. POSTMASTER: send address changes to Pacific Journal of Mathematics, P.O. Box 4163, Berkeley, CA 94704-0163.

PJM peer review and production are managed by EditFLOW ${ }^{\mathrm{TM}}$ from Mathematical Sciences Publishers.

PUBLISHED BY PACIFIC JOURNAL OF MATHEMATICS

at the University of California, Berkeley 94720-3840

A NON-PROFIT CORPORATION

Typeset in LATEX

Copyright $(02012$ by Pacific Journal of Mathematics 


\section{PACIFIC JOURNAL OF MATHEMATICS}

Volume $255 \quad$ No. $1 \quad$ January 2012

Averaging sequences

FERnANDo Alcalde Cuesta and Ana Rechtman

Affine group schemes over symmetric monoidal categories

ABHISHEK BANERJEE

Eigenvalue estimates on domains in complete noncompact Riemannian

manifolds

Daguang Chen, TAO Zheng and Min LU

Realizing the local Weil representation over a number field

GERALD CLIFF and DAVID MCNEILLY

Lagrangian submanifolds in complex projective space with parallel second fundamental form

Franki DiLlen, Haizhong Li, LuC VRANCKEN and XiANFENG

WANG

Ultra-discretization of the $D_{4}^{(3)}$-geometric crystal to the $G_{2}^{(1)}$-perfect crystals

Mana Igarashi, KaIlash C. MisRa and ToshiKi NAKASHIMA Connectivity properties for actions on locally finite trees

KEITH JONES

Remarks on the curvature behavior at the first singular time of the Ricci flow

NAM Q. LE and NATASA SESUm

Stability of capillary surfaces with planar boundary in the absence of gravity

PetKo I. MARINOV

Small hyperbolic polyhedra

SHAWN RAFALSKI

Hurwitz spaces of coverings with two special fibers and monodromy group 241 a Weyl group of type $B_{d}$

FRANCESCA VETRO 